\title{
Roller discharge of thin film filter cakes from membranes: A key to the thin film filtration
}

\author{
Zihim Lam*, Harald Anlauf, Hermann Nirschl \\ Institute of Mechanical Process Engineering and Mechanics (MVM), Karlsruhe Institute of Technology (KIT), Straße am Forum 8, 76131 Karlsruhe, Germany
}

Keywords:

Filter cake discharge

Thin film filtration

Roller discharge

Pasty filter cake

\begin{abstract}
A B S T R A C T
A novel continuous process for the mechanical separation of very difficult-to-filter suspensions is the thin film filtration. Highly compressible materials build a thin layer of particles on the filter medium with a high porosity gradient. The first much more compressed cake layer reduces the filtrate flow drastically and the following filtration becomes less efficient. The thin film filtration technology is based on forming a thin filter cake and an immediate discharge of the filter after dewatering to maintain a high product throughput. However the discharge of the thin filter cake is still a challenge. Here we present a new method to discharge the thin filter cake using a roller. The experiments on the discharge behavior of the roller are carried out on a filter plate using yeast as a model material. As a result the discharge in an opposite direction of rotation removed the filter cake completely and even reduced the residual moisture without damaging the filter medium.
\end{abstract}

\section{Introduction}

For the separation of fine particles such as pigments, microalgae, yeasts or other biological and organic particles there are several equipments available today such as centrifuges, crossflow filters, dis continuous pressure filters or continuous precoat vacuum drum filters $\left[\begin{array}{ll}1 & 7\end{array}\right]$. Centrifuges show weakness when the density difference between the particles and the surrounding medium is low. In this case the re quired energy to separate them would be very high. More than $50 \%$ of the energy consumption for example in a decanter centrifuge is used for the acceleration of the suspension [8]. For the crossflow filtration a higher residual moisture content is inevitable to ensure a flowable re tentate. Whereas the pressure filter can achieve a filter cake with a low residual moisture, but highly compressible particles cause a very long filtration time, which reduces its efficiency. A precoat filtration on a vacuum drum filter might be a good solution for the filtration of compressible particle, but comes along with other drawbacks like the contamination with the precoat material and high consumption of precoat material $[9,10]$. A promising filtration technology for highly compressible materials is the thin film filtration on a vacuum drum filter. It uses microporous membranes for the direct filtration of these particles and eliminates the risk of the contamination with precoat material. The filter cake of the very compressible particles has a high porosity gradient. The layer near the membrane has the lowest porosity which will significantly slow down the following filtration $[11,12]$.
Therefore this layer of particles with a high filter resistance and a low porosity needs to be discharged to maintain a high filtration efficiency. However the filter cake discharge of very thin filter cakes on a vacuum drum filter is still a challenge for the novel thin film filtration [13]. For the vacuum drum filter there are several commonly used discharge methods available [14]. The scraper discharge is the most commonly used method for a drum filter. But a scraper is not suitable for the discharge of very thin filter cakes since an exact circularity of the drum filter is necessary to scrape off the thin filter cake and to prevent the scraper from destroying the membrane. Another option is the endless belt discharge which lifts the filter media from the vacuum drum filter and carries it to a discharge roll, followed by an aligning roll and re turns to the drum filter. However this system requires a good me chanical stress resistance of the filter media [15]. Since the novel thin film filtration is using membranes with a lower tensile stress resistance, this discharge method is also not appropriate. There are roller discharge methods, which are currently used to remove pasty filter cakes, but they show some other disadvantages. These roller discharge methods use a comb to scratch the filter cake from the roller leaving one part of the filter cake on the roller to pick up the filter cake on the drum filter. Hence, the unique property of the filter cake to stick to themselves is necessary for this method to maintain a proper discharge. Therefore a new discharge method is needed for the discharge of pasty and thin filter cakes, which even have a low cohesion in addition.

In this paper we investigate how a roller discharge in an opposite

\footnotetext{
* Corresponding author.

E-mail address: Zihim.Lam@kit.edu (Z. Lam).
} 
moving direction can discharge a filter cake from a filter plate. First, the different parameters in the same direction of movement of the roller are examined and the proportions of the filter cake on the roller are com pared. The operating parameters affecting the discharge behavior like the applied force on the roller and the gas differential pressure, which prevents the filter cake from rewetting during the discharge are in vestigated. The rollers with different diameters and Shore A hardnesses are tested as well and evaluated with regard to the proportion of the filter cake on the roller. Then, these results are compared to the roller discharge in an opposite moving direction. At last we discuss the in fluence of the diameter, the Shore A hardness and the moving direction on the proportion of the discharged cake and on its residual moisture.

\section{Material and methods}

\subsection{Sample preparation and characterization}

As a model system commercially available wet baker's yeast Saccharomyces cerevisiae with a mean particle size $x_{50,3}$ of $5.9 \mu \mathrm{m}$ was purchased from FALA GmbH (Kehl, Germany). The particle size was measured with laser diffraction (HELOS/Quixel, Sympatec GmbH, Germany). The yeast was suspended in $1 \mathrm{~g} / \mathrm{L}$ dextrose, $1 \mathrm{mM}$ phosphate buffer and $1 \mathrm{~g} / \mathrm{L}$ bacteriological peptone and had a dry weight of $25 \mathrm{~g} /$ L. The $\mathrm{pH}$ of 6.5 of the suspension was measured using a $\mathrm{pH}$ meter (WTW pH 3310, Xylem Analytics Germany GmbH, Weilheim, Germany). To determine the dry weight the yeast was dried in an oven for $24 \mathrm{~h}$ at a temperature of $90 \pm 5^{\circ} \mathrm{C}$.

\subsection{Experimental setup}

For the cake formation, a filter plate with a filter area $A_{P}$ of $(6 \times 12)$ $\mathrm{cm}^{2}=72 \mathrm{~cm}^{2}$ as shown in Fig. 1a was used. The pressure difference on

a)

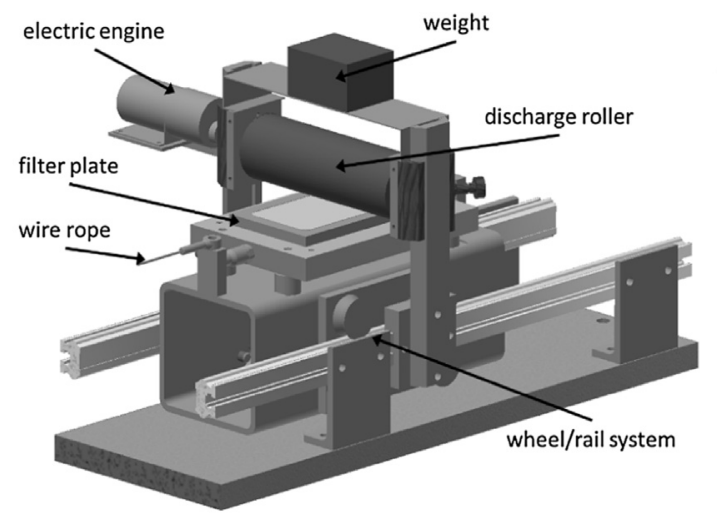

b)

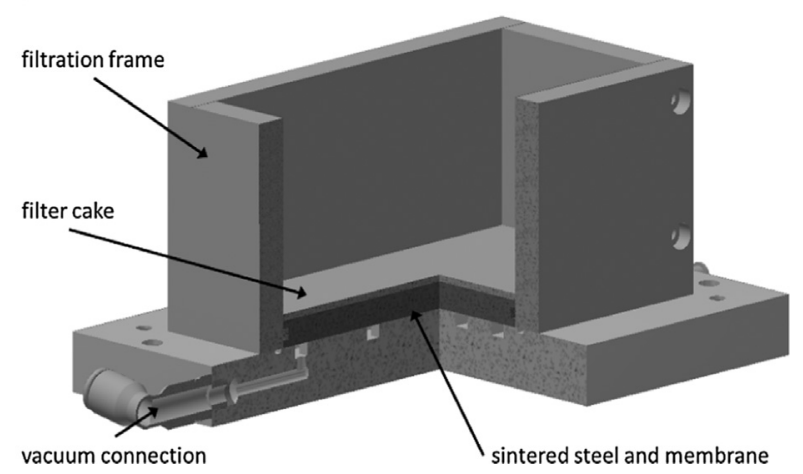

Fig. 1. (a) The experimental setup of the roller discharger and (b) the filter plate with a filtration frame. the filter plate filter was generated by a vacuum pump (UNO 6, Pfeiffer Vacuum GmbH, Asslar, Germany). A track etched membrane with supporting fibers made of polyethylene terephthalate (PET) and an average pore size of $1 \mu \mathrm{m}$ was used (RoTrac, OXYPHEN AG, Wetzikon, Switzerland). The membrane was supported by a stainless steel sintered material (Siperm R14, Tridelta Siperm GmbH, Dortmund, Germany). The height of the filter cake was measured by a laser displacement sensor (LK G157, Keyence Deutschland GmbH, Neu Isenburg, Ger many). The roller discharger with an aluminum core for the filter plate and for the vacuum drum filter was coated with a $5 \mathrm{~mm}$ layer of ac rylonitrile butadiene rubber (NBR) with different Shore A hardnesses. The measurements of the Shore A hardness were carried out using a shore durometer (SAUTER HBA 1000 durometer, Kern \& Sohn GmbH, Balingen Frommern, Germany). Investigations of the roller discharge behaviour on the filter plate were conducted with the experimental setup shown in Fig. 1a. The filter plate with the membrane is fixed on a wheel rail system which is pulled by a wire rope. The wire rope is connected to a computer aided traction machine to set the desired speed of the filter plate (FRANK 81813, Karl Frank GmbH, Weinheim Birkenau, Germany). The rotational speed of the discharge roller can be adjusted by an electric engine (RS Pro 940D DC, RS Components GmbH, Mörfelden Walldorf, Germany). To adjust the line load of the roller on the plate there are different weights on top of the roller. The line load $q_{P}$ is calculated from the weight $m$, the gravitational force $g$ and the width $L_{P}$ of the filter plate (Eq. (1)):

$q_{P}=\frac{m \cdot g}{L_{P}}$

In this experimental setup of the filter plate the different roller diameters of $60 \mathrm{~cm}, 90 \mathrm{~cm}$, and $110 \mathrm{~cm}$ can be installed for each series of experiments.

\subsection{Experimental procedure}

For the analysis of the roller discharge behavior on the filter plate each filter cake was built at $80 \mathrm{kPa}$ and discharged at the related gas differential pressure to prevent the rewetting of the filter cake. The yeast suspension was filled in a filtration frame on the filter plate as shown in Fig. 1b, which was removed after the cake was build. The filter cake height, which was kept constant at $0.25 \mathrm{~mm}$ for all the ex periments was measured using the laser displacement sensor. The filter plate including the filter cake was mounted on the wheel rail system shown in Fig. 1a and was pulled by a wire rope at a constant speed. For the first set of experiments the roller was rotating in the same moving direction as the plate resulting in a relative speed of $v_{\text {rel }}=0 \mathrm{~mm} / \mathrm{min}$ between the filter plate and the roller. The second set of experiments discharged the cake at the opposite direction of rotation, resulting in a relative speed of $v_{r e l}=400 \mathrm{~mm} / \mathrm{min}$. The relative speed $v_{\text {rel }}$ of these two sets of experiments is calculated from the speed of the filter plate $v_{\text {plate }}$, the rotational speed $s_{\text {rot }}$ and the radius $r$ of the roller in equation (2):

$v_{\text {rel }}=v_{\text {plate }}-\left(r \cdot s_{\text {rot }}\right)$

To determine the proportion of the discharged filter cake Q, the cake on the roller as well as the remaining cake on the membrane were dried in an oven at a temperature of $90 \pm 5{ }^{\circ} \mathrm{C}$ for $24 \mathrm{~h}$. The proportion of the discharged cake $\mathrm{Q}$ was calculated from the dry mass of the dis charged cake on the membrane $m_{M}$ and the total dry mass of the formed cake $m_{\text {tot }}$ as shown in Eq. (3).

$Q=\frac{m_{t o t}-m_{M}}{m_{\text {tot }}} \cdot 100 \%$

The residual moisture RM was calculated from the total mass of the wet filter cake $m_{\text {tot,wet }}$ and the dry mass of the filter cake $m_{\text {tot }}$ as seen in Eq. (4): 
a)

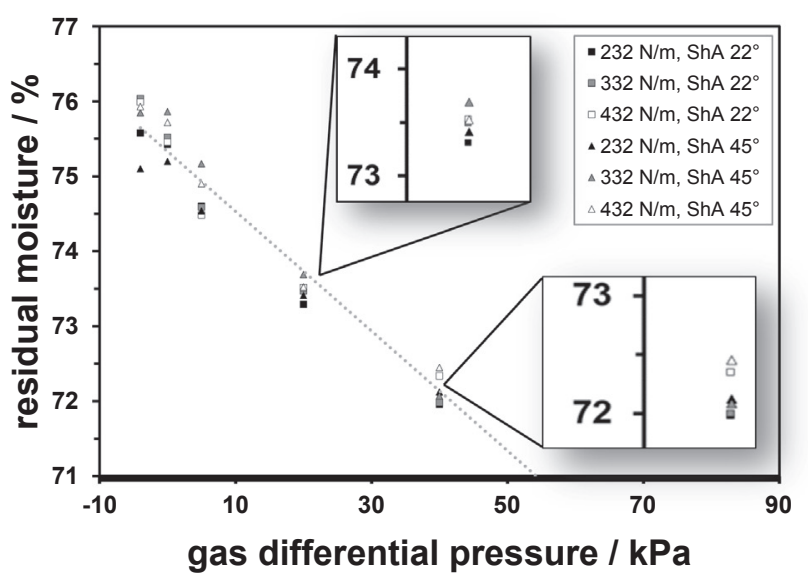

b)

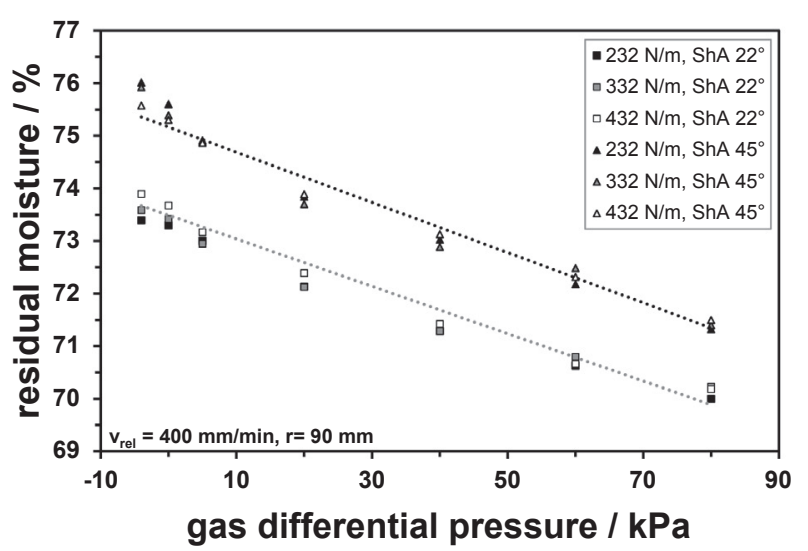

Fig. 2. Residual moisture of the filter cake discharged at the same (a) and opposite (b) direction of rotation on the filter plate (Shore A hardness = ShA).

$R M=\frac{m_{t o t, w e t}-m_{t o t}}{m_{t o t, w e t}} \cdot 100 \%$

Since the yeast cells have intracellular fluids the residual moisture is higher than particles without intracellular fluids.

\section{Results and discussion}

\subsection{Residual moisture at different directions of rotation}

The discharge was performed while a gas differential pressure was applied. The residual moisture depends on the gas differential pressure during the discharge. Fig. 2 illustrates the residual moisture at different directions of rotation of the roller discharger, at different line loads and

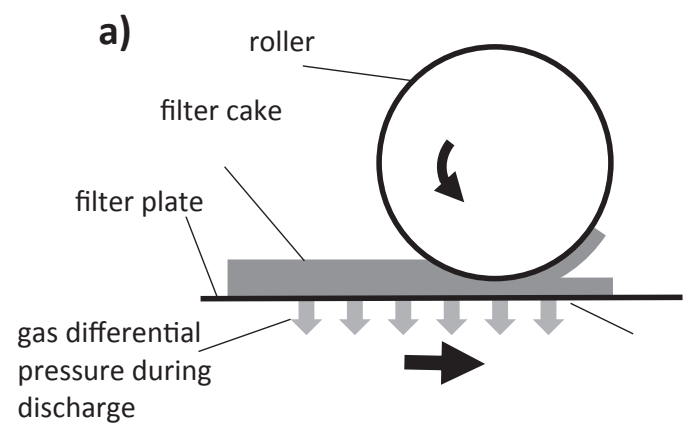

at different Shore A hardnesses on the filter plate.

In both cases the residual moisture of the filter cake decreases with a higher gas differential pressure. For the discharge at the same direction of rotation the Shore A hardness of $22^{\circ}$ and $45^{\circ}$ have a similar residual moisture (see Fig. 2a). The experiments in the opposite direction of rotation show that a roller with a lower Shore A hardness reduces the residual moisture of the filter cake. By comparing The experimental results of a roller with a Shore A hardness of $22^{\circ}$ in Fig. $2 \mathrm{~b}$ in the op posite direction of rotation and a roller with a Shore A hardness of $22^{\circ}$ in Fig. 2a in the same direction of rotation, the cake is significantly drier when using the opposite discharge direction. There is evidence which shows the impact of the shear rate and pressure on the final dry solid fraction [16 19]. Hereby the cake experiences a shear force caused by the opposite discharge method, which rearranges the cake structure and releases the pore liquid, resulting in a lower residual moisture. The released liquid is sucked by the membrane since the gas differential pressure is applied during the discharge. Except the two values at $0 \mathrm{kPa}$ and $-5 \mathrm{kPa}$ are discharged at a negative differential pressure which results in a slightly higher residual moisture due to the rewetting of the filter cake. Whereas the cake discharged in the same direction of ro tation is only stressed by the vertical pressure of the roller causing a higher residual moisture than the opposite discharge method. The line load of $232 \mathrm{~N} / \mathrm{m}, 332 \mathrm{~N} / \mathrm{m}$ and $432 \mathrm{~N} / \mathrm{m}$ has a low influence on the residual moisture of each set of experiments and a higher line load didn't lead to a further noticeable change of the residual moisture. Above a gas differential pressure of $40 \mathrm{kPa}$ the amount of the dis charged filter cake was too low to evaluate the results. Therefore no values of the residual moisture are available for these process para meters. The scheme in Fig. 3a and b shows the two different directions of rotation.

\subsection{Discharge behavior of the roller in the same direction of rotation on the filter plate}

The direction of rotation of the roller is a significant factor for the discharge behavior. In the following the influence of the diameter, the Shore A hardness and the line load of the roller in the same direction of rotation will be presented. A discharge in the same direction of rotation is affected by the adhesive force between the roller and the filter cake, the cohesion of the filter cake, the adhesion between the filter cake and the membrane. When the adhesive force between the filter cake and the membrane is higher than the adhesion between the filter cake and the roller the discharge of the filter cake becomes incomplete. Once the adhesive force between the filter cake and the roller is higher than the adhesive force between the filter cake and the membrane, the propor tion of the discharged cake $Q$ is higher. Another important factor is the cohesion of the filter cake. If the cohesion of the filter cake is low the filter cake will break apart in two halves, which results in a partly discharge of the filter cake. The applied pressure during the discharge increases the adhesive force between the filter cake and the membrane. As it can be seen in Fig. 4 the proportion of the filter cake left on the

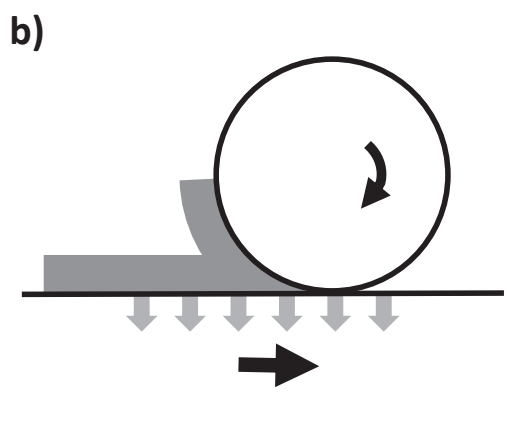

Fig. 3. Schemes of the discharge (a) in the same direction of rotation at $\mathrm{v}_{\text {rel }}=0 \mathrm{~mm} / \mathrm{min}$ and (b) in the opposite direction of rotation at $\mathrm{v}_{\text {rel }}=400 \mathrm{~min} / \mathrm{min}$. 


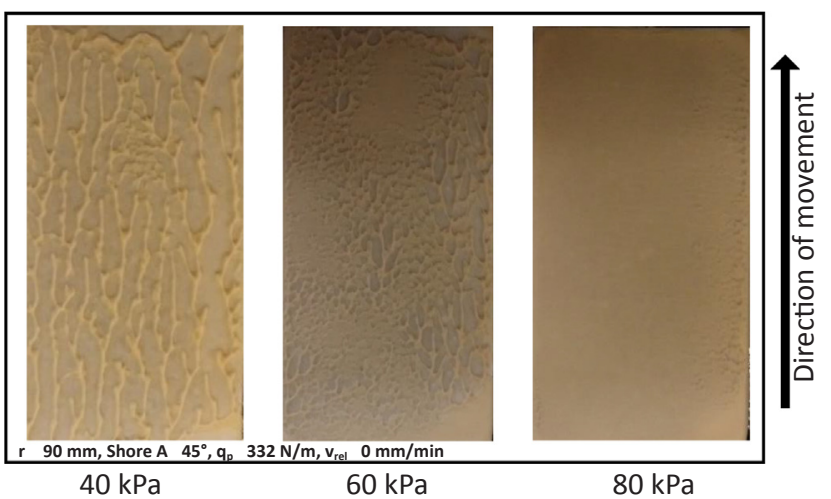

Fig. 4. Influence of the gas differential pressure during the discharge on the proportion of the filter cake.

membrane increases with a higher applied gas differential pressure during the discharge. Furthermore the discharge at $40 \mathrm{kPa}$ shows stripes along the direction of movement of the filter plate. These stripes be come less with an increasing gas differential pressure during the dis charge due to the stronger adhesive force between the membrane and the cake. This effect can be observed in all experiments with the same direction of rotation.

Other influence parameter of the discharge are shown in Fig. 5. In Fig. 5 a the effect of the diameter of the roller on the proportion of the discharged cake is illustrated. The larger diameter of the roller increases the proportion of the discharged cake. The larger diameter causes a different contact angle between the filter plate and the roller. The contact angle decreases with an increasing diameter resulting in a better uptake and therefore increased discharge of the filter cake. Whereas the line load as shown in Fig. $5 \mathrm{~b}$ has a little effect on the proportion of the discharged cake. The proportion of the discharged cake at the line load 232, 332 and $432 \mathrm{~N} / \mathrm{m}$ have a similar result. The increasing Shore A hardness of the roller doesn't show a significant trend (Fig. 5 c). The three influence parameters don't allow a complete discharge of the filter cake, which would affect the following filtration efficiency.

\subsection{Discharge behavior of the roller in the opposite direction of rotation on the filter plate}

The discharge in the opposite direction of rotation has a significant effect on the proportion of the discharged cake. Fig. 6 a and Fig. 6 b show a complete discharge of the filter cake using a roller with a Shore A hardness of $22^{\circ}$. The diameter of $110 \mathrm{~mm}, 90 \mathrm{~mm}$, and $60 \mathrm{~mm}$ as well as the line load of $432 \mathrm{~N} / \mathrm{m}, 332 \mathrm{~N} / \mathrm{m}$ and $232 \mathrm{~N} / \mathrm{m}$ did not influence the result of the complete discharge. In contrast to the discharge in the same direction of rotation, the contact angle between the filter plate and the roller has no effect on the cake discharge. The apparent increase of the discharged cake can be explained by the shear stress caused by the opposite direction of rotation of the roller. But is notable that a higher Shore A hardness as shown in Fig. $6 \mathrm{c}$ reduces the proportion of the discharged cake. In this case even the higher gas differential pres sure, which is applied during the discharge decreases the proportion of the discharged cake. Therefore a softer roller is more suitable for the stable operation of the cake discharge in an opposite direction of ro tation.

In the opposite direction of rotation the filter cake moves towards the roller and builds up a cluster in front of it. The cluster is then re moved by the rotation of the roller as it can be seen in Fig. 7 .

The accumulation of the filter cake in front of the roller results in a stripe pattern on the roller after the discharge. The stripe patterns are depicted in Fig. 8.

As shown in Fig. 9 one main criterion for the discharge in an

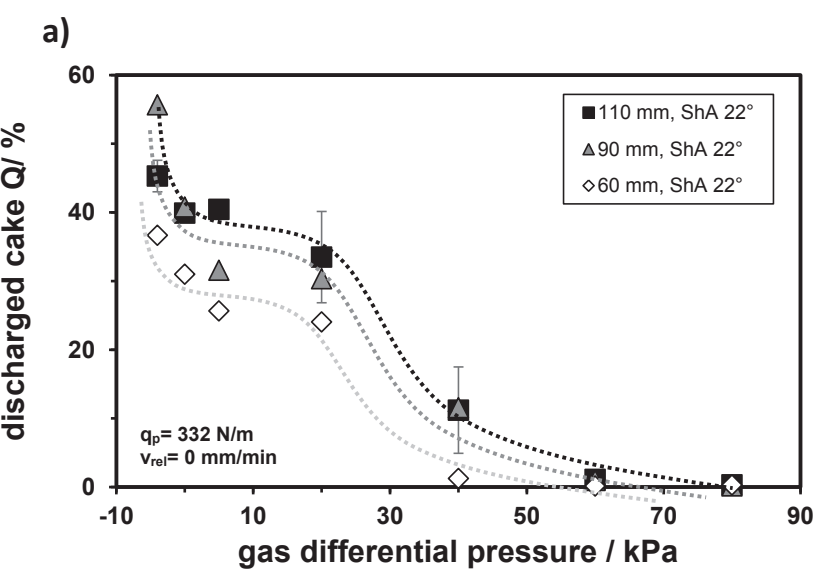

b)

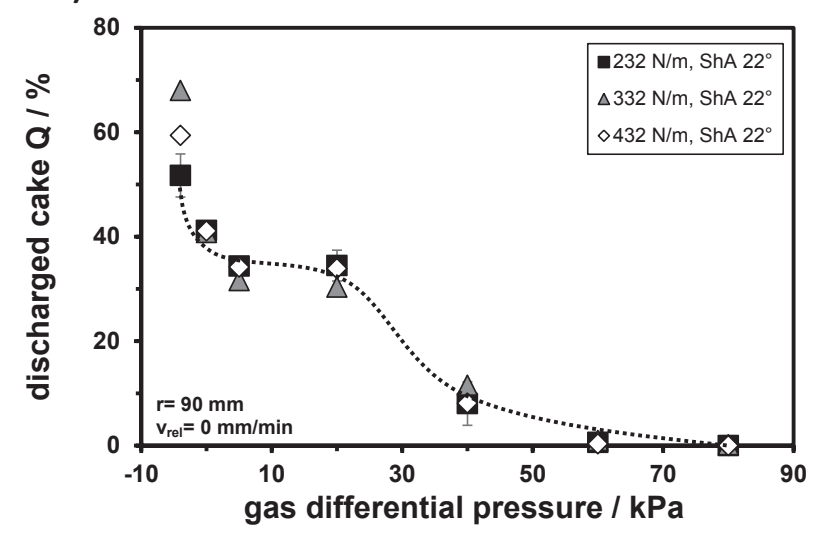

c)

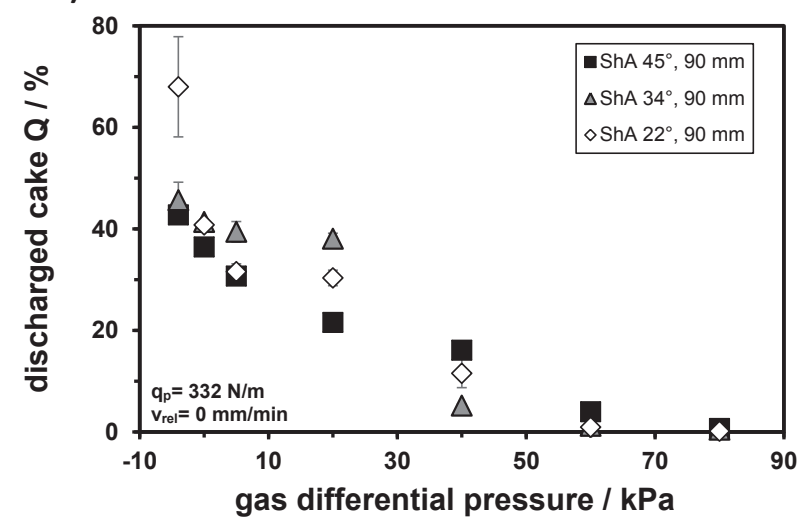

Fig. 5. Influence of the different roller diameter (a), the line load (b) and the Shore A hardness on the proportion of the discharged cake (c) in the same direction of rotation.

opposite direction of rotation is the relative speed $v_{r e l}$ between the roller and the filter plate. The filter cake accumulates in front of the roller at a low relative speed and slips below the roller when the cluster becomes too large. At a higher relative speed the cluster is removed by the faster rotation of the roller and prevents the formation of a large cluster. Therefore less filter cake can slip underneath the roller and increases the proportion of the discharged filter cake.

\section{Conclusion}

The roller discharge behavior of very thin and pasty filter cakes from membranes was analyzed. Two operational methods were in vestigated: the rotation of the roller in the same and opposite direction of rotation using rollers with different Shore A hardnesses, diameters 
a)

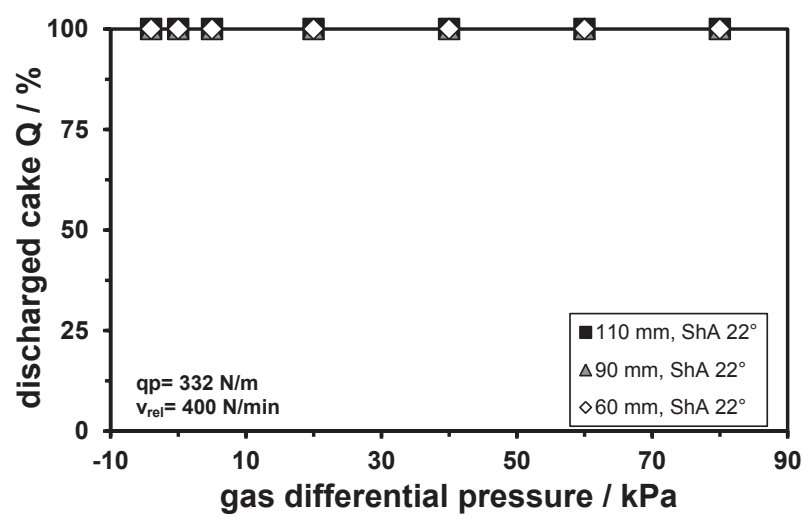

b)

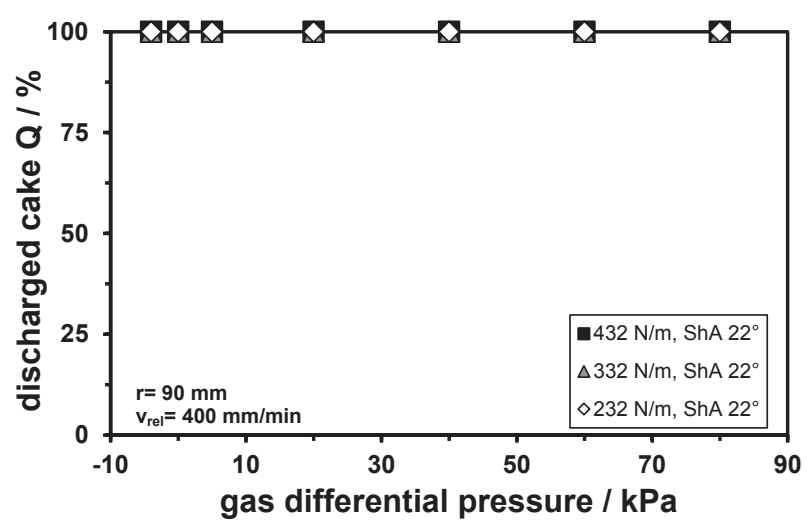

c)

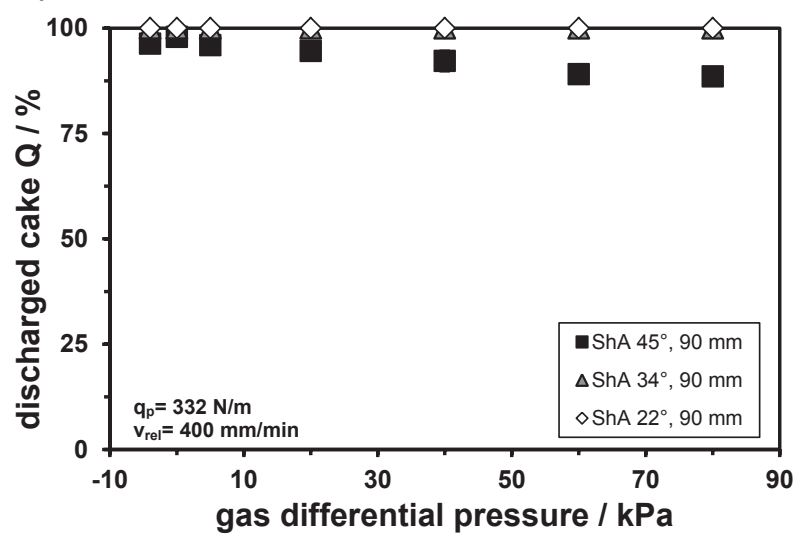

Fig. 6. Influence of the different roller diameter (a), the line load (b) and the Shore A hardness on the proportion of the discharged cake (c) in the opposite direction of rotation.

and line loads. The filter cake splits in two halves once the roller and the filter plate move in the same direction and the proportion of the discharged filter cake increases with a larger diameter of the roller. With regard to the utilization in an industrial process this method would not be recommended, since the discharge is incomplete.

Whereas the roller discharge in an opposite direction of rotation was found to be an effective way to completely remove pasty and thin filter cakes from membranes. The shear force of the roller which affects the filter cake rearranges the filter cake structure causing the leakage of inner liquid. A gas differential pressure applied during the discharge removes the liquid and the residual moisture of the filter cake is lower. This discharge method appears to be a promising method for the thin film filtration to remove thin and pasty filter cakes from membranes

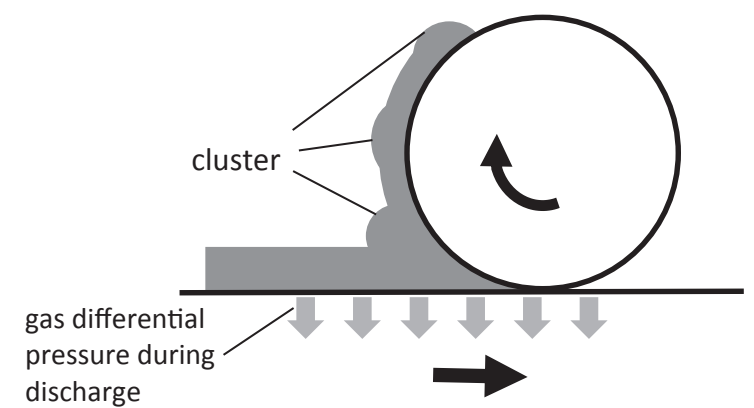

Fig. 7. A cluster builds up in front of the roller and is removed by the opposite rotation of the roller.

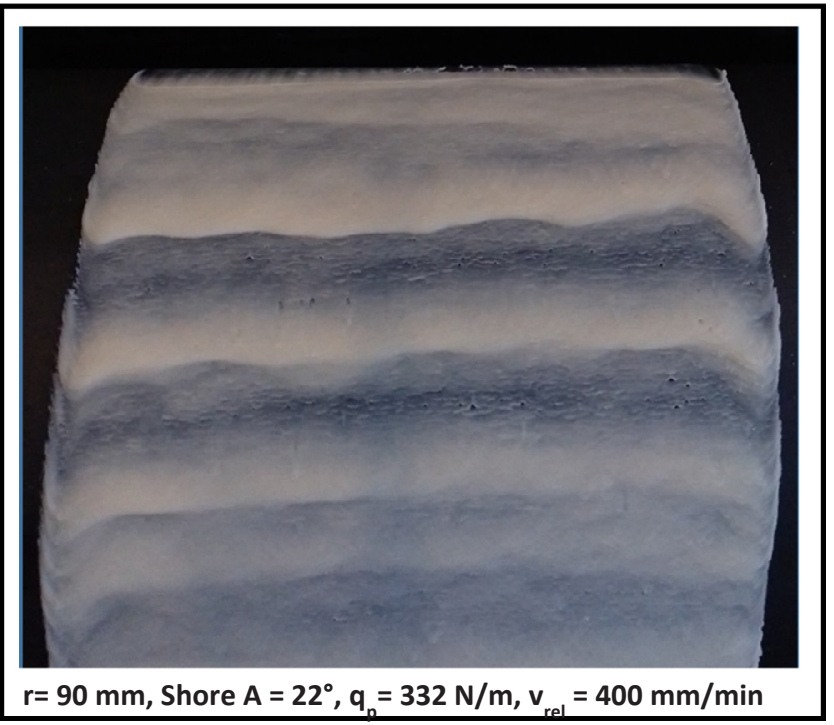

Fig. 8. The clusters of the discharged filter cake form horizontal stripe patterns on the roller.

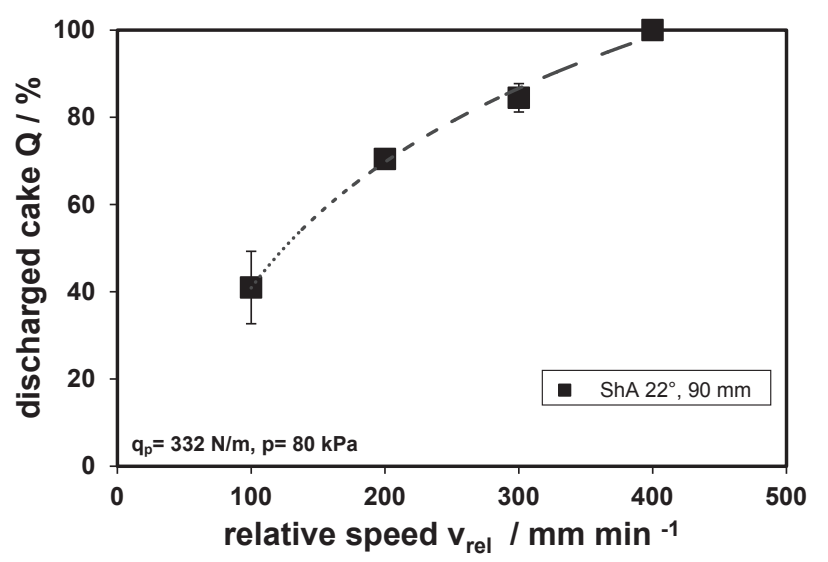

Fig. 9. The proportion of the discharged filter cake increases at a higher relative speed $v_{\text {rel }}$.

and comes with a further reduction of the residual moisture of the filter cake.

\section{Funding}

The author would like to thank AiF for the funding of the project $18603 \mathrm{~N}$. 


\section{Acknowledgments}

The authors would like to thank H. Stobitzer for performing parts of the experiments and R. Ullrich for the construction of the experimental setup.

\section{References}

[1] E. Molina Grima, E. Belarbi, F.G. Acien Fernandez, A. Medina, Y. Chisti, Recovery of microalgal biomass and metabolites: process options and economics, Biotechnol. Adv. 20 (7-8) (2003) 491-515.

[2] L.E. Spelter, A. Steiwand, H. Nirschl, Processing of dispersions containing fine particles or biological products in tubular bowl centrifuges, Chem. Eng. Sci. 65 (2010) 4173-4181.

[3] M. Konrath, M. Hackbarth, H. Nirschl, Process monitoring and control for constant separation conditions in centrifugal classification of fine particles, Adv. Powder Technol. 25 (2014) 991-998.

[4] H. Anlauf, Recent developments in centrifuge technology, Sep. Purif. Technol. (2007), https://doi.org/10.1016/j.seppur.2007.05.012.

[5] A.I. Barros, A.L. Gonçalves, M. Simões, J.C.M. Pires, Harvesting techniques applied to microalgae: a review, Renew. Sustain. Energy Rev. 41 (2015) 1489-1500.

[6] C. Boittelle, C. Poupot, Vladan Milisic, M. Mietton-Peuchot, Advances in the precoat filtration process, Sep. Sci. Technol. 43 (7) (2008) 1701-1712.

[7] N. Rossignol, L. Vandanjon, P. Jaouen, F. Quéméneur, Membrane technology for the continuous separation microalgae/culture medium: compared performances of cross-flow microfiltration and ultrafiltration, Aquacult. Eng. 20 (1999) 191-208.
[8] J. Hermeler, L. Horstkötter, T. Hartmann, Neue Dekantergeneration mit verbessertem energetischen Wirkungsgrad, F\&S Filtrieren und Separieren 26 (3) (2012) $158-166$.

[9] F. Braun, W. Back, T. Becker, M. Krottenthaler, Entwicklung eines regenerierbaren Filterhilfsmittels auf Zellulosebasis, Abschlußbericht Az 24962, Deutsche Bundesstiftung Umwelt, Germany, 2010.

[10] T. Jung, Kieselgurfreie Anschwemmfiltration, Der Deutsche Weinbau, Scientific report, 27, Germany, 2013.

[11] F. Tiller, T. Green, Role of porosity in filtration IX skin effect with highly com pressible materials, AIChe J. 19 (6) (1973) 1266

12] F.M. Tiller, H. Cooper, Role of porosity infiltration: Part V. Porosity variation in filter cakes, AIChe J. 33 (8) (1962) 445.

[13] H. Anlauf, A. Erk, Continuous, skin "Filtration of Very Difficult-to-Filter Suspensions, Aufbereitungs-Technik 47 (3) (2006) 22-29.

[14] C. Cox, F. Traczyk, Design features and types of filtration equipment, Soc. Mining Metall. Explor. 2 (2002) 1346

[15] R.J. Wakeman, Separation technologies for sludge dewatering, J. Hazard. Mater. 144 (3) (2007) 614-619.

[16] J. Vaxelaire, J. Olivier, Compression dewatering of particulate suspensions and sludge: effect of shear, Drying Technol. 32 (1) (2014) 23

[17] M.A. Koenders, E. Liebhart, R.J. Wakeman, DEAD-END filtration with torsional shear: experimental findings and theoretical analysis, Trans. Icheme 79 (2001) 249.

[18] R.J. Wakeman, G.M. Zhang, M.A. Koenders, Effects of rotational flow induced in a rotary piston press on the formation and properties of filter cakes, in: Proceedings of World Filtration Congress, 2000, 1121.

[19] F.S. Lau, M.C. Mensinger, M.J. Roberts, Development and testing of the internal rotary compression press, Drying Technol. 8 (1) (1990) 1-23. 
Karlsruher Institut für Technologie

\section{Repository KITopen}

Dies ist ein Postprint/begutachtetes Manuskript.

Empfohlene Zitierung:

Lam, Z.; Anlauf, H.; Nirschl, H.

Roller discharge of thin film filter cakes from membranes: A key to the thin film filtration. 2019. Separation and purification technology, 221.

doi: $\underline{10.5445 / / R / 1000094427}$

Zitierung der Originalveröffentlichung:

Lam, Z.; Anlauf, H.; Nirschl, H.

Roller discharge of thin film filter cakes from membranes: A key to the thin film filtration. 2019. Separation and purification technology, 221, 38-43.

doi:10.1016/j.seppur.2019.03.062

Lizenzinformationen: CC BY-NC ND Lizenz 4.0 